\title{
Regularly $\left(\mathscr{I}_{2}, \mathscr{I}\right)$-Convergence and Regularly $\left(\mathscr{I}_{2}, \mathscr{I}\right)$-Cauchy Double Sequences of Fuzzy Numbers
}

\author{
Erdinç Dündar, ${ }^{1}$ Özer Talo, ${ }^{2}$ and Feyzi Başar ${ }^{3}$ \\ ${ }^{1}$ Department of Mathematics, Kocatepe University, Gazligöl Yolu, 03200 Afyon, Turkey \\ ${ }^{2}$ Department of Mathematics, Celal Bayar University, 45040 Manisa, Turkey \\ ${ }^{3}$ Department of Mathematics, Fatih University, The Hadimköy Campus, Büyükçekmece, 34500 Istanbul, Turkey
}

Correspondence should be addressed to Feyzi Başar; feyzibasar@gmail.com

Received 1 November 2012; Accepted 28 February 2013

Academic Editor: Malte Braack

Copyright (C) 2013 Erdinç Dündar et al. This is an open access article distributed under the Creative Commons Attribution License, which permits unrestricted use, distribution, and reproduction in any medium, provided the original work is properly cited.

In this paper, we introduce the notions of regularly $\left(\mathscr{I}_{2}, \mathscr{I}\right),\left(\mathscr{I}_{2}^{*}, \mathscr{I}^{*}\right)$-convergence and regularly $\left(\mathscr{I}_{2}, \mathscr{I}\right),\left(\mathscr{J}_{2}^{*}, \mathscr{I}^{*}\right)$-Cauchy double sequence of fuzzy numbers. Also, we study some properties of these concepts.

\section{Introduction, Notations, and Definitions}

The concept of ordinary convergence of a sequence of fuzzy numbers was firstly introduced by Matloka [1] and proved some basic theorems for sequences of fuzzy numbers. Nanda [2] studied the sequences of fuzzy numbers and showed that the set of all convergent sequences of fuzzy numbers forms a complete metric space. Recently, Nuray and Savaş [3] defined the concepts of statistical convergence and statistical Cauchy for sequence of fuzzy numbers. They proved that a sequence of fuzzy numbers, is statistically convergent if and only if it is statistically Cauchy. Nuray [4] introduced Lacunary statistical convergence of sequences of fuzzy numbers whereas Savaş [5] studied some equivalent alternative conditions for a sequence of fuzzy numbers to be statistically Cauchy. A lot of developments have been made in this area after the works of [6-8].

Throughout the paper $\mathbb{N}$ and $\mathbb{R}$ denote the set of all positive integers and the set of all real numbers, respectively. The idea of $\mathscr{I}$-convergence was introduced by Kostyrko et al. [9] as a generalization of statistical convergence which is based on the structure of the ideal $\mathscr{I}$ of subset of the set $\mathbb{N}$. Nuray and Ruckle [10] independently introduced the same with another name generalized statistical convergence. Das et al. [11] introduced the concept of $\mathscr{I}$-convergence of double sequences in a metric space and studied some properties of this convergent sequences of this type. Balcerzak et al.
[12] studied on statistical convergence and ideal convergence for sequences of functions. Komisarski [13] discussed the pointwise $\mathscr{I}$-convergence and $\mathscr{I}$-convergence in measure of sequences of functions. Mursaleen and Alotaibi investigated the notion of ideal convergence in [14] for random 2-normed space and construct some interesting examples. Mursaleen and Mohiuddine defined and studied the concept of $\mathscr{I}$ convergence, $\mathscr{I}^{*}$-convergence, $\mathscr{I}$-limit points and $\mathscr{I}$-cluster points in probabilistic normed space, in [15]. Şahiner et al. introduced and investigated $\mathscr{I}$-convergence in 2-normed spaces, and also defined and examined some new sequence spaces using norm, in [16]. A lot of developments have been made in this area after the works of [17-21].

V. Kumar and K. Kumar studied the concepts of $\mathscr{I}$ convergence, $\mathscr{I}^{*}$-convergence, and $\mathscr{I}$-Cauchy sequence for sequences of fuzzy numbers, in [22]. Mursaleen et al. studied the concept of ideal convergence and ideal Cauchy for double sequences in intuitionistic fuzzy normed spaces, in [23]. Recently, Dündar and Talo have introduced the concepts of $\mathscr{I}_{2}$-convergence, $\mathscr{I}_{2}^{*}$-convergence for double sequences of fuzzy numbers and studied their some properties and relations, in [24]. Quite recently, Dündar and Talo have introduced the concepts of $\mathscr{I}_{2}$-Cauchy, $\mathscr{I}_{2}^{*}$-Cauchy double sequences of fuzzy numbers, in [25].

In this paper, we introduce the notions of regularly $\left(\mathscr{I}_{2}, \mathscr{I}\right),\left(\mathscr{I}_{2}^{*}, \mathscr{I}^{*}\right)$-convergence and regularly $\left(\mathscr{I}_{2}, \mathscr{I}\right)$, 
$\left(\mathscr{I}_{2}^{*}, \mathscr{I}^{*}\right)$-Cauchy double sequence of fuzzy numbers. Also, we study some properties of those sequences.

In a possible application we can say that if we choose the statistical convergence of a special case of the ideal convergence, the results in this paper can be obtained for statistical convergence.

Now, we recall the concept of fuzzy numbers, convergence, ideal convergence of the sequences and double sequences (see $[9,11,21,24-30]$ ).

A double sequence $x=\left(x_{m n}\right)_{m, n \in \mathbb{N}}$ of real numbers is said to be convergent to $L \in \mathbb{R}$ in Pringsheim's sense, if for any $\varepsilon>0$ there exists $N_{\varepsilon} \in \mathbb{N}$ such that $\left|x_{m n}-L\right|<\varepsilon$ whenever $m, n>N_{\varepsilon}$. In this case we write

$$
P-\lim _{m, n \rightarrow \infty} x_{m n}=L \quad \text { or } \quad \lim _{m, n \rightarrow \infty} x_{m n}=L .
$$

Let $X \neq \emptyset$. A class $\mathscr{I}$ of subsets of $X$ is said to be an ideal in $X$ provided that

(i) $\emptyset \in \mathscr{I}$,

(ii) $A, B \in \mathscr{I}$ implies $A \cup B \in \mathscr{I}$,

(iii) $A \in \mathscr{I}, B \subset A$ implies $B \in \mathscr{I}$.

$\mathscr{I}$ is called a nontrivial ideal if $X \notin \mathscr{I}$.

Let $X \neq \emptyset$. A non-empty class $\mathscr{F}$ of subsets of $X$ is said to be a filter in $X$ provided that

(i) $\emptyset \notin \mathscr{F}$,

(ii) $A, B \in \mathscr{F}$ implies $A \cap B \in \mathscr{F}$,

(iii) $A \in \mathscr{F}, A \subset B$ implies $B \in \mathscr{F}$.

If $\mathscr{I}$ is a nontrivial ideal in $X, X \neq \emptyset$, then the class

$$
\mathscr{F}(\mathscr{I})=\{M \subset X:(\exists A \in \mathscr{I})(M=X \backslash A)\}
$$

is called a filter on $X$ associated with $\mathscr{I}$.

A nontrivial ideal $\mathscr{I}$ in $X$ is called admissible if $\{x\} \in \mathscr{I}$ for each $x \in X$.

Throughout the paper we take $\mathscr{I}$ as a nontrivial admissible ideal in $\mathbb{N}$.

Let $\mathscr{I} \subset 2^{\mathbb{N}}$ be a nontrivial ideal, and let $(X, \rho)$ be a metric space. A sequence $\left(x_{n}\right)$ of elements of $X$ is said to be $\mathscr{I}$-convergent to $L \in X$, if for each $\varepsilon>0$ we have $A(\varepsilon)=\{n \in$ $\left.\mathbb{N}: \rho\left(x_{n}, L\right) \geq \varepsilon\right\} \in \mathscr{I}$.

A sequence $\left(x_{n}\right)$ of elements of $X$ is said to be $\mathscr{J}^{*}$-convergent to $L \in X$ if and only if there exists a set $M \in \mathscr{F}(\mathscr{I})$ (i.e., $\mathbb{N} \backslash M \in \mathscr{I}), M=\left\{m_{1}<m_{2}<\cdots<m_{k}<\cdots\right\}$ such that $\rho\left(x_{m_{k}}, L\right) \rightarrow 0$, as $k \rightarrow \infty$.

Throughout the paper we take $\mathscr{I}_{2}$ as a nontrivial admissible ideal in $\mathbb{N} \times \mathbb{N}$.

A nontrivial ideal $\mathscr{I}_{2} \subset 2^{\mathbb{N} \times \mathbb{N}}$ is called strongly admissible if $\{i\} \times \mathbb{N}$ and $\mathbb{N} \times\{i\}$ belong to $\mathscr{I}_{2}$ for each $i \in \mathbb{N}$. It is evident that a strongly admissible ideal is also admissible.

Let $\mathscr{I}_{2}^{0}=\{A \subset \mathbb{N} \times \mathbb{N}:(\exists m(A) \in \mathbb{N})(i, j \geq m(A) \Rightarrow$ $(i, j) \notin A)\}$. Then $\mathscr{I}_{2}^{0}$ is a nontrivial strongly admissible ideal and clearly an ideal $\mathscr{I}_{2}$ is strongly admissible if and only if $\mathscr{I}_{2}^{0} \subset \mathscr{I}_{2}$.

Let $(X, \rho)$ be a linear metric space, and let $\mathscr{I}_{2} \subset 2^{\mathbb{N} \times \mathbb{N}}$ be a strongly admissible ideal. A double sequence $x=\left(x_{m n}\right)$ in
$X$ is said to be $\mathscr{I}_{2}$-convergent to $L \in X$, if for any $\varepsilon>0$ we have $A(\varepsilon)=\left\{(m, n) \in \mathbb{N} \times \mathbb{N}: \rho\left(x_{m n}, L\right) \geq \varepsilon\right\} \in \mathscr{I}_{2}$ and is written $\mathscr{I}_{2}-\lim _{m, n \rightarrow \infty} x_{m n}=L$.

If $\mathscr{I}_{2} \subset 2^{\mathbb{N} \times \mathbb{N}}$ is a strongly admissible ideal, then usual convergence implies $\mathscr{I}_{2}$-convergence.

Let $(X, \rho)$ be a linear metric space, and let $\mathscr{I}_{2} \subset 2^{\mathbb{N} \times \mathbb{N}}$ be a strongly admissible ideal. A double sequence $x=\left(x_{m n}\right)$ of elements of $X$ is said to be $\mathscr{I}_{2}^{*}$-convergent to $L \in X$, if and only if there exists a set $M \in \mathscr{F}\left(\mathscr{I}_{2}\right)$ (i.e., $\mathbb{N} \times \mathbb{N} \backslash M \in \mathscr{I}_{2}$ ) such that $\lim _{m, n \rightarrow \infty} x_{m n}=L$, for $(m, n) \in M$ and is written $\mathscr{I}_{2}^{*}-\lim _{m, n \rightarrow \infty} x_{m n}=L$.

Let $(X, \rho)$ be a linear metric space, and let $\mathscr{I}_{2} \subset 2^{\mathbb{N} \times \mathbb{N}}$ be a strongly admissible ideal. A double sequence $x=\left(x_{m n}\right)$ of elements of $X$ is said to be $\mathscr{I}_{2}$-Cauchy, if for every $\varepsilon>0$ there exist $s=s(\varepsilon), t=t(\varepsilon) \in \mathbb{N}$ such that $A(\varepsilon)=\{(m, n) \in \mathbb{N} \times \mathbb{N}$ : $\left.\rho\left(x_{m n}, x_{s t}\right) \geq \varepsilon\right\} \in \mathscr{I}_{2}$.

Let $\mathscr{I}_{2}$ be an ideal of $\mathbb{N} \times \mathbb{N}$ and $\mathscr{I}$ be an ideal of $\mathbb{N}$, then a double sequence $x=\left(x_{m n}\right)$ in $\mathbb{C}$, which is the set of complex numbers, is said to be regularly $\left(\mathscr{I}_{2}, \mathscr{I}\right)$-convergent $\left(r\left(\mathscr{I}_{2}, \mathscr{I}\right)\right.$-convergent), if it is $\mathscr{I}_{2}$-convergent in Pringsheim's sense and for every $\varepsilon>0$, the following statements hold:

$$
\left\{m \in \mathbb{N}:\left|x_{m n}-L_{n}\right| \geq \varepsilon\right\} \in \mathscr{I}
$$

for some $L_{n} \in \mathbb{C}$, for each $n \in \mathbb{N}$ and

$$
\left\{n \in \mathbb{N}:\left|x_{m n}-K_{m}\right| \geq \varepsilon\right\} \in \mathscr{I}
$$

for some $K_{m} \in \mathbb{C}$, for each $m \in \mathbb{N}$.

We say that an admissible ideal $\mathscr{I} \subset 2^{\mathbb{N}}$ satisfies the property (AP), if for every countable family of mutually disjoint sets $\left\{A_{1}, A_{2}, \ldots\right\}$ belonging to $\mathscr{F}$, there exists a countable family of sets $\left\{B_{1}, B_{2}, \ldots\right\}$ such that $A_{j} \triangle B_{j}$ is a finite set for $j \in \mathbb{N}$ and $B=\bigcup_{j=1}^{\infty} B_{j} \in \mathscr{I}$. (hence $B_{j} \in \mathscr{I}$ for each $j \in \mathbb{N}$ ).

We say that an admissible ideal $\mathscr{I}_{2} \subset 2^{\mathbb{N} \times \mathbb{N}}$ satisfies the property (AP2), if for every countable family of mutually disjoint sets $\left\{A_{1}, A_{2}, \ldots\right\}$ belonging to $\mathscr{I}_{2}$, there exists a countable family of sets $\left\{B_{1}, B_{2}, \ldots\right\}$ such that $A_{j} \triangle B_{j} \in \mathscr{I}_{2}^{0}$, that is, $A_{j} \triangle B_{j}$ is included in the finite union of rows and columns in $\mathbb{N} \times \mathbb{N}$ for each $j \in \mathbb{N}$ and $B=\bigcup_{j=1}^{\infty} B_{j} \in \mathscr{I}_{2}$ (hence $B_{j} \in \mathscr{I}_{2}$ for each $j \in \mathbb{N}$ ).

A fuzzy number is a fuzzy set on the real axis, that is, a mapping $u: \mathbb{R} \rightarrow[0,1]$ which satisfies the following four conditions.

(i) $u$ is normal, that is, there exists an $x_{0} \in \mathbb{R}$ such that $u\left(x_{0}\right)=1$.

(ii) $u$ is fuzzy convex, that is, $u[\lambda x+(1-\lambda) y] \geq$ $\min \{u(x), u(y)\}$ for all $x, y \in \mathbb{R}$ and for all $\lambda \in[0,1]$.

(iii) $u$ is upper semicontinuous.

(iv) The set $[u]_{0}:=\overline{\{x \in \mathbb{R}: u(x)>0\}}$ is compact, (cf. Zadeh [31]), where $\overline{\{x \in \mathbb{R}: u(x)>0\}}$ denotes the closure of the set $\{x \in \mathbb{R}: u(x)>0\}$ in the usual topology of $\mathbb{R}$. 
We denote the set of all fuzzy numbers on $\mathbb{R}$ by $E^{1}$ and called it as the space of fuzzy numbers. $\alpha$-level set $[u]_{\alpha}$ of $u \in E^{1}$ is defined by

$$
[u]_{\alpha}:= \begin{cases}\{t \in \mathbb{R}: x(t) \geq \alpha\}, & (0<\alpha \leq 1), \\ \{t \in \mathbb{R}: x(t)>\alpha\}, & (\alpha=0) .\end{cases}
$$

The set $[u]_{\alpha}$ is closed, bounded, and nonempty interval for each $\alpha \in[0,1]$ which is defined by $[u]_{\alpha}:=\left[u^{-}(\alpha), u^{+}(\alpha)\right]$. $\mathbb{R}$ can be embedded in $E^{1}$, since each $r \in \mathbb{R}$ can be regarded as a fuzzy number $\bar{r}$ defined by

$$
\bar{r}(x):= \begin{cases}1, & (x=r), \\ 0, & (x \neq r) .\end{cases}
$$

Theorem 1 (see [27]). Let $[u]_{\alpha}=\left[u^{-}(\alpha), u^{+}(\alpha)\right]$ for $u \in E^{1}$ and for each $\alpha \in[0,1]$. Then the following statements hold.

(i) $u^{-}$is a bounded and nondecreasing left continuous function on $(0,1]$.

(ii) $u^{+}$is a bounded and nonincreasing left continuous function on $(0,1]$.

(iii) The functions $u^{-}$and $u^{+}$are right continuous at the point $\alpha=0$.

(iv) $u^{-}(1) \leq u^{+}(1)$.

Conversely, if the pair of functions $\alpha$ and $\beta$ satisfies the conditions (i)-(iv), then there exists a unique $u \in E^{1}$ such that $[u]_{\alpha}:=\left[u^{-}(\alpha), u^{+}(\alpha)\right]$ for each $\alpha \in[0,1]$. The fuzzy number $u$ corresponding to the pair of functions $u^{-}$and $u^{+}$is defined by $u: \mathbb{R} \rightarrow[0,1], u(x):=\sup \left\{\alpha: u^{-}(\alpha) \leq x \leq u^{+}(\alpha)\right\}$.

Let $u, v, w \in E^{1}$ and $k \in \mathbb{R}$. Then the operations addition, scalar multiplication, and product are defined on $E^{1}$ by

$$
\begin{gathered}
u+v=w \Longleftrightarrow[w]_{\alpha}=[u]_{\alpha}+[v]_{\alpha}, \quad \forall \alpha \in[0,1] \\
\Longleftrightarrow w^{-}(\alpha)=u^{-}(\alpha)+v^{-}(\alpha), \\
w^{+}(\alpha)=u^{+}(\alpha)+v^{+}(\alpha), \\
{[k u]_{\alpha}=k[u]_{\alpha}, \quad \forall \alpha \in[0,1],} \\
u v=w \Longleftrightarrow[w]_{\alpha}=[u]_{\alpha}[v]_{\alpha}, \quad \forall \alpha \in[0,1],
\end{gathered}
$$

where it is immediate that

$$
\begin{gathered}
w^{-}(\alpha)=\min \left\{u^{-}(\alpha) v^{-}(\alpha), u^{-}(\alpha) v^{+}(\alpha), u^{+}(\alpha) v^{-}(\alpha),\right. \\
\left.u^{+}(\alpha) v^{+}(\alpha)\right\}, \\
w^{+}(\alpha)=\max \left\{u^{-}(\alpha) v^{-}(\alpha), u^{-}(\alpha) v^{+}(\alpha), u^{+}(\alpha) v^{-}(\alpha),\right. \\
\left.u^{+}(\alpha) v^{+}(\alpha)\right\},
\end{gathered}
$$

for all $\alpha \in[0,1]$.
Let $W$ be the set of all closed bounded intervals $A$ of real numbers with endpoints $\underline{A}$ and $\bar{A}$, that is, $A:=[\underline{A}, \bar{A}]$. Define the relation $d$ on $W$ by

$$
d(A, B):=\max \{|\underline{A}-\underline{B}|,|\bar{A}-\bar{B}|\} .
$$

Then it can easily be observed that $d$ is a metric on $W$ and $(W, d)$ is a complete metric space, (cf. Nanda [2]). Now, we may define the metric $D$ on $E^{1}$ by means of the Hausdorff metric $d$ as

$$
\begin{aligned}
D(u, v) & :=\sup _{\alpha \in[0,1]} d\left([u]_{\alpha},[v]_{\alpha}\right) \\
& :=\sup _{\alpha \in[0,1]} \max \left\{\left|u^{-}(\alpha)-v^{-}(\alpha)\right|,\left|u^{+}(\alpha)-v^{+}(\alpha)\right|\right\} .
\end{aligned}
$$

One can see that

$$
\begin{aligned}
D(u, \overline{0}) & =\sup _{\alpha \in[0,1]} \max \left\{\left|u^{-}(\alpha)\right|,\left|u^{+}(\alpha)\right|\right\} \\
& =\max \left\{\left|u^{-}(0)\right|,\left|u^{+}(0)\right|\right\} .
\end{aligned}
$$

The partial ordering relation $\preceq$ on $E^{1}$ is defined as follows:

$$
u \leq v \Longleftrightarrow u^{-}(\alpha) \leq v^{-}(\alpha), \quad u^{+}(\alpha) \leq v^{+}(\alpha) \quad \forall \alpha \in[0,1] .
$$

Now, we may give the following.

Proposition 2 (see [6]). Let $u, v, w, z \in E^{1}$ and $k \in \mathbb{R}$. Then, the following statements hold.

(i) $\left(E^{1}, D\right)$ is a complete metric space.

(ii) $D(k u, k v)=|k| D(u, v)$.

(iii) $D(u+v, w+v)=D(u, w)$.

(iv) $D(u+v, w+z) \leq D(u, w)+D(v, z)$.

(v) $|D(u, \overline{0})-D(v, \overline{0})| \leq D(u, v) \leq D(u, \overline{0})+D(v, \overline{0})$.

Following Matloka [1], we give some definitions concerning the sequences of fuzzy numbers which are needed in the text.

A sequence $u=\left(u_{k}\right)$ of fuzzy numbers is a function $u$ from the set $\mathbb{N}$ into the set $E^{1}$. The fuzzy number $u_{k}$ denotes the value of the function at $k \in \mathbb{N}$ and is called as the general term of the sequence. By $w(F)$, we denote the set of all sequences of fuzzy numbers

A sequence $\left(u_{n}\right) \in w(F)$ is called convergent with limit $u \in E^{1}$, if for every $\varepsilon>0$ there exists an $n_{0}=n_{0}(\varepsilon) \in \mathbb{N}$ such that $D\left(u_{n}, u\right)<\varepsilon$ for all $n \geq n_{0}$.

A double sequence $u=\left(u_{n k}\right)$ of fuzzy real numbers is defined by a function $u$ from the set $\mathbb{N} \times \mathbb{N}$ into the set $E^{1}$. The fuzzy number $u_{n k}$ denotes the value of the function at $(n, k) \in \mathbb{N} \times \mathbb{N}$.

A double sequence $u=\left(u_{m n}\right)$ of fuzzy numbers is said to be convergent in the Pringsheim's sense or P-convergent, if for every $\varepsilon>0$ there exists $k \in \mathbb{N}$ such that $D\left(u_{m n}, u_{0}\right)<\varepsilon$ for all $m, n \geq k$ and is denoted by $P-\lim _{m, n \rightarrow \infty} u_{m n}=u_{0}$. The fuzzy number $u_{0}$ is called the Pringsheim limit of $u$. 
Let $\mathscr{I}_{2} \subset 2^{\mathbb{N} \times \mathbb{N}}$ be a strongly admissible ideal.

(i) A double sequence $u=\left(u_{m n}\right)$ of fuzzy numbers is said to be $\mathscr{I}_{2}$-convergent to a fuzzy number $u_{0}$ if for any $\varepsilon>0$ we have $A(\varepsilon)=\left\{(m, n) \in \mathbb{N} \times \mathbb{N}: D\left(u_{m n}, u_{0}\right) \geq\right.$ $\varepsilon\} \in \mathscr{I}_{2}$ and is written $\mathscr{I}_{2}-\lim _{m, n \rightarrow \infty} u_{m n}=u_{0}$.

(ii) A double sequence $u=\left(u_{m n}\right)$ of fuzzy numbers is said to be $\mathscr{I}_{2}^{*}$-convergent to $u_{0} \in E^{1}$ if there exists $M \in$ $\mathscr{F}\left(\mathscr{I}_{2}\right)$ (i.e., $H=\mathbb{N} \times \mathbb{N} \backslash M \in \mathscr{I}_{2}$ ) such that

$$
\lim _{m, n \rightarrow \infty} u_{m n}=u_{0}
$$

and is written $\mathscr{I}_{2}^{*}-\lim _{m, n \rightarrow \infty} u_{m n}=u_{0}$.

(iii) A double sequence $u=\left(u_{m n}\right)$ of fuzzy numbers is said to be $\mathscr{I}_{2}$-Cauchy, if for each $\varepsilon>0$, there exist $s=$ $s(\varepsilon), t=t(\varepsilon) \in \mathbb{N}$ such that $A(\varepsilon)=\{(m, n) \in \mathbb{N} \times \mathbb{N}$ : $\left.D\left(u_{m n}, u_{s t}\right) \geq \varepsilon\right\} \in \mathscr{I}_{2}$.

(iv) A double sequence $u=\left(u_{m n}\right)$ of fuzzy numbers is said to be $\mathscr{I}_{2}^{*}$-Cauchy if there exists a set $M \in \mathscr{F}\left(\mathscr{I}_{2}\right)$ (i.e., $H=\mathbb{N} \times \mathbb{N} \backslash M \in \mathscr{I}_{2}$ ) such that for every $\varepsilon>0$ and for $(m, n),(s, t) \in M, m, n, s, t>k_{0}=k_{0}(\varepsilon), D\left(u_{m n}, u_{s t}\right)<$ $\varepsilon$, that is,

$$
\lim _{\substack{m, n, s, t \rightarrow \infty \\(m, n),(s, t) \in M}} D\left(u_{m n}, u_{s t}\right)=0 .
$$

Now, we begin with quoting the following five lemmas due to Dündar and Talo $[24,25]$ which are needed throughout the paper.

Lemma 3 (see [24, Theorem 3.3]). Let $\mathscr{I}_{2} \subset 2^{\mathbb{N} \times \mathbb{N}}$ be a strongly admissible ideal, let $u=\left(u_{m n}\right)$ be a double sequence of fuzzy numbers, and let $u_{0}$ be a fuzzy number. Then, $P$ $\lim _{m, n \rightarrow \infty} u_{m n}=u_{0}$ implies $\mathscr{I}_{2}-\lim _{m, n \rightarrow \infty} u_{m n}=u_{0}$.

Lemma 4 (see [24, Theorem 4.2]). Let $\mathscr{I}_{2} \subset 2^{\mathbb{N} \times \mathbb{N}}$ be a strongly admissible ideal, let $u=\left(u_{m n}\right)$ be a double sequence of fuzzy numbers, and let $u_{0} \in E^{1}$. Then, $\mathscr{I}_{2}^{*}-\lim _{m, n \rightarrow \infty} u_{m n}=$ $u_{0}$ implies $\mathscr{I}_{2}-\lim _{m, n \rightarrow \infty} u_{m n}=u_{0}$.

Lemma 5 (see [24, Theorem 4.4]). Let $\mathscr{I}_{2} \subset 2^{\mathbb{N} \times \mathbb{N}}$ be a strongly admissible ideal with property (AP2), let $u=\left(u_{m n}\right)$ be a double sequence of fuzzy numbers, and let $u_{0}$ be a fuzzy real number. Then, $\mathscr{I}_{2}-\lim _{m, n \rightarrow \infty} u_{m n}=u_{0}$ implies $\mathscr{I}_{2}^{*}-$ $\lim _{m, n \rightarrow \infty} u_{m n}=u_{0}$.

Lemma 6 (see [25, Theorem 3.2]). Let $\mathscr{I}_{2} \subset 2^{\mathbb{N} \times \mathbb{N}}$ be a strongly admissible ideal. A double sequence $u=\left(u_{m n}\right)$ of fuzzy numbers is $\mathscr{I}_{2}$-convergent if and only if it is $\mathscr{I}_{2}$-Cauchy sequence.

Lemma 7 (see [25, Theorem 3.4]). Let $\mathscr{I}_{2} \subset 2^{\mathbb{N} \times \mathbb{N}}$ be a strongly admissible ideal. If a double sequence $u=\left(u_{m n}\right)$ of fuzzy numbers is an $\mathscr{I}_{2}^{*}$-Cauchy sequence, then it is $\mathscr{I}_{2}$-Cauchy.

\section{Main Results}

In this section, we study certain properties of regular convergence, regularly $\left(\mathscr{I}_{2}, \mathscr{I}\right)$-convergence and regularly $\left(\mathscr{I}_{2}, \mathscr{I}\right)$ Cauchy double sequences of fuzzy numbers.

Definition 8. A double sequence $\left(u_{m n}\right)$ of fuzzy numbers is said to be regularly convergent, if it is convergent in Pringsheim's sense and the limits

$$
\lim _{m \rightarrow \infty} u_{m n}, \quad(n \in \mathbb{N}), \quad \lim _{n \rightarrow \infty} u_{m n}, \quad(m \in \mathbb{N}),
$$

exist for each fixed $n \in \mathbb{N}$ and $m \in \mathbb{N}$, respectively. Note that if $\left(u_{m n}\right)$ is regularly convergent to a fuzzy number $u_{0}$, then the limits

$$
\lim _{n \rightarrow \infty} \lim _{m \rightarrow \infty} u_{m n}, \quad \lim _{m \rightarrow \infty} \lim _{m \rightarrow \infty} u_{m n}
$$

exist and are equal to $u_{0}$. In this case we write

$$
r-\lim _{m, n \rightarrow \infty} u_{m n}=u_{0} \quad \text { or } \quad u_{m n} \stackrel{r}{\longrightarrow} u_{0} \quad \text { as } m, n \rightarrow \infty \text {. }
$$

Definition 9. Let $\mathscr{I}_{2} \subset 2^{\mathbb{N} \times \mathbb{N}}$ be a strongly admissible ideal, and let $\mathscr{I} \subset 2^{\mathbb{N}}$ be an admissible ideal. A double sequence $\left(u_{m n}\right)$ of fuzzy numbers is said to be regularly $\left(\mathscr{I}_{2}, \mathscr{I}\right)$ convergent $\left(r\left(\mathscr{I}_{2}, \mathscr{I}\right)\right.$-convergent $)$, if it is $\mathscr{I}_{2}$-convergent in Pringsheim's sense and for every $\varepsilon>0$, the following statements hold:

$$
\left\{m \in \mathbb{N}: D\left(u_{m n}, v_{n}\right) \geq \varepsilon\right\} \in \mathscr{I}
$$

for some fuzzy numbers $v_{n}$, for each $n \in \mathbb{N}$ and

$$
\left\{n \in \mathbb{N}: D\left(u_{m n}, w_{m}\right) \geq \varepsilon\right\} \in \mathscr{I}
$$

for some fuzzy numbers $w_{m}$, for each $m \in \mathbb{N}$.

In the case $\left(u_{m n}\right)$ is regularly $\left(\mathscr{I}_{2}, \mathscr{I}\right)$-convergent $\left(r\left(\mathscr{I}_{2}, \mathscr{I}\right)\right.$-convergent) to a fuzzy number $u_{0}$, then the limits $\mathscr{I}-\lim _{n \rightarrow \infty} \lim _{m \rightarrow \infty} u_{m n}$ and $\mathscr{I}-\lim _{m \rightarrow \infty} \lim _{n \rightarrow \infty} u_{m n}$ exist and are equal to $u_{0}$.

Theorem 10. Let $\mathscr{I}_{2}$ be a strongly admissible ideal of $\mathbb{N} \times \mathbb{N}$, and let $\mathscr{I}$ be an admissible ideal of $\mathbb{N}$. If a double sequence $\left(u_{m n}\right)$ of fuzzy numbers is regularly convergent, then it is $r\left(\mathscr{I}_{2}, \mathscr{I}\right)$-convergent.

Proof. Let $\left(u_{m n}\right)$ be regularly convergent. Then $\left(u_{m n}\right)$ is convergent in Pringsheim's sense and the limits $\lim _{m \rightarrow \infty} u_{m n}(n \in$ $\mathbb{N})$ and $\lim _{n \rightarrow \infty} u_{m n}(m \in \mathbb{N})$ exist. By Lemma 3, $\left(u_{m n}\right)$ is $\mathscr{I}_{2}$-convergent. Also, for $\varepsilon>0$, there exist $m=m_{0}(\varepsilon)$ and $n=n_{0}(\varepsilon)$ such that

$$
D\left(u_{m n}, v_{n}\right)<\varepsilon
$$

for some fuzzy numbers $v_{n}$ and each fixed $n \in \mathbb{N}$ for every $m \geq m_{0}$ and

$$
D\left(u_{m n}, w_{m}\right)<\varepsilon
$$


for some fuzzy numbers $w_{m}$ and each fixed $m \in \mathbb{N}$ for every $n \geq n_{0}$. Then, since $\mathscr{I}$ is admissible ideal so for $\varepsilon>0$, we have

$$
\begin{aligned}
& \left\{m \in \mathbb{N}: D\left(u_{m n}, v_{n}\right) \geq \varepsilon\right\}=\left\{1,2, \ldots, m_{0}-1\right\} \in \mathscr{I}, \\
& \left\{n \in \mathbb{N}: D\left(u_{m n}, w_{m}\right) \geq \varepsilon\right\}=\left\{1,2, \ldots, n_{0}-1\right\} \in \mathscr{I} .
\end{aligned}
$$

Hence, $\left(u_{m n}\right)$ is $r\left(\mathscr{I}_{2}, \mathscr{I}\right)$-convergent.

Definition 11. Let $\mathscr{I}_{2}$ be a strongly admissible ideal of $\mathbb{N} \times$ $\mathbb{N}$, and let $\mathscr{I}$ be an admissible ideal of $\mathbb{N}$. A double sequence $\left(u_{m n}\right)$ of fuzzy numbers is said to be $r\left(\mathscr{I}_{2}^{*}, \mathscr{I}^{*}\right)$-convergent, if there exist the sets $M \in \mathscr{F}\left(\mathscr{I}_{2}\right)$ (i.e., $\left.\mathbb{N} \times \mathbb{N} \backslash M \in \mathscr{I}_{2}\right), M_{1} \in$ $\mathscr{F}(\mathscr{I})$, and $M_{2} \in \mathscr{F}(\mathscr{I})$ (i.e., $\mathbb{N} \backslash M_{1} \in \mathscr{I}$ and $\mathbb{N} \backslash M_{2} \in \mathscr{I}$ ) such that the limits

$$
\lim _{\substack{m, n \rightarrow \infty \\(m, n) \in M}} u_{m n}, \quad \lim _{\substack{m \rightarrow \infty \\ m \in M_{1}}} u_{m n}, \quad \lim _{\substack{n \rightarrow \infty \\ n \in M_{2}}} u_{m n}
$$

exist for each fixed $n \in \mathbb{N}$ and $m \in \mathbb{N}$, respectively.

Theorem 12. Let $\mathscr{I}_{2}$ be a strongly admissible ideal of $\mathbb{N} \times \mathbb{N}$, and let $\mathscr{I}$ be an admissible ideal of $\mathbb{N}$. If a double sequence $\left(u_{m n}\right)$ of fuzzy numbers is $r\left(\mathscr{I}_{2}^{*}, \mathscr{I}^{*}\right)$-convergent, then it is $r\left(\mathscr{I}_{2}, \mathscr{I}\right)$-convergent.

Proof. Let $\left(u_{m n}\right)$ be $r\left(\mathscr{I}_{2}^{*}, \mathscr{I}^{*}\right)$-convergent. Then, it is $\mathscr{I}_{2}^{*}$ convergent and so, by Lemma 4 , it is $\mathscr{I}_{2}$-convergent. Also, there exist the sets $M_{1}, M_{2} \in \mathscr{F}(\mathscr{I})$ such that

$$
\begin{aligned}
& (\forall \varepsilon>0) \quad\left(\exists m_{0} \in \mathbb{N}\right) \quad\left(\forall m \geq m_{0}\right) \quad\left(m \in M_{1}\right) \\
& D\left(u_{m n}, v_{n}\right)<\varepsilon, \quad(n \in \mathbb{N})
\end{aligned}
$$

for some fuzzy numbers $v_{n}$ and

$$
\begin{aligned}
& (\forall \varepsilon>0) \quad\left(\exists n_{0} \in \mathbb{N}\right) \quad\left(\forall n \geq n_{0}\right) \quad\left(n \in M_{2}\right) \\
& D\left(u_{m n}, w_{m}\right)<\varepsilon, \quad(m \in \mathbb{N})
\end{aligned}
$$

for some fuzzy numbers $w_{m}$. Hence, we have

$$
\begin{array}{r}
A(\varepsilon)=\left\{m \in \mathbb{N}: D\left(u_{m n}, v_{n}\right) \geq \varepsilon\right\} \subset H_{1} \cup\left\{1,2, \ldots, m_{0}-1\right\}, \\
(n \in \mathbb{N}), \\
B(\varepsilon)=\left\{n \in \mathbb{N}: D\left(u_{m n}, w_{m}\right) \geq \varepsilon\right\} \subset H_{2} \cup\left\{1,2, \ldots, n_{0}-1\right\},
\end{array}
$$

for $H_{1}, H_{2} \in \mathscr{I}$. Since $\mathscr{I}$ is admissible ideal we get

$$
\begin{aligned}
& H_{1} \cup\left\{1,2, \ldots,\left(m_{0}-1\right)\right\} \in \mathscr{I}, \\
& H_{2} \cup\left\{1,2, \ldots, n_{0}-1\right\} \in \mathscr{I},
\end{aligned}
$$

and therefore $A(\varepsilon), B(\varepsilon) \in \mathscr{I}$. This shows that the double sequence $\left(u_{m n}\right)$ is $r\left(\mathscr{I}_{2}, \mathscr{I}\right)$-convergent.

Theorem 13. Let $\mathscr{I}_{2} \subset 2^{\mathbb{N} \times \mathbb{N}}$ be a strongly admissible ideal with property (AP2), and let $\mathscr{I} \subset 2^{\mathbb{N}}$ be an admissible ideal with property (AP). If a double sequence $\left(u_{m n}\right)$ of fuzzy numbers is $r\left(\mathscr{I}_{2}, \mathscr{I}\right)$-convergent, then $\left(u_{m n}\right)$ is $r\left(\mathscr{I}_{2}^{*}, \mathscr{I}^{*}\right)$ convergent.
Proof. Let a double sequence $\left(u_{m n}\right)$ of fuzzy numbers be $r\left(\mathscr{I}_{2}, \mathscr{I}\right)$-convergent. Then $\left(u_{m n}\right)$ is $\mathscr{I}_{2}$-convergent and so $\left(u_{m n}\right)$ is $\mathscr{I}_{2}^{*}$-convergent, by Lemma 5 . Also, for every $\varepsilon>0$ we have

$$
A(\varepsilon)=\left\{m \in \mathbb{N}: D\left(u_{m n}, v_{n}\right) \geq \varepsilon\right\} \in \mathscr{I}
$$

for some fuzzy numbers $v_{n}$, for each $n \in \mathbb{N}$ and

$$
C(\varepsilon)=\left\{n \in \mathbb{N}: D\left(u_{m n}, w_{m}\right) \geq \varepsilon\right\} \in \mathscr{I}
$$

for some fuzzy numbers $w_{m}$, for each $m \in \mathbb{N}$.

Now put

$$
\begin{aligned}
& A_{1}=\left\{m \in \mathbb{N}: D\left(u_{m n}, v_{n}\right) \geq 1\right\}, \\
& A_{k}=\left\{m \in \mathbb{N}: \frac{1}{k} \leq D\left(u_{m n}, v_{n}\right)<\frac{1}{k-1}\right\}
\end{aligned}
$$

for $k \geq 2$, for some fuzzy numbers $v_{n}$ and for each $n \in \mathbb{N}$. It is clear that $A_{i} \cap A_{j}=\emptyset$ for $i \neq j$ and $A_{i} \in \mathscr{I}$ for each $i \in \mathbb{N}$. By the property $(A P)$ there is a countable family of sets $\left\{B_{1}, B_{2}, \ldots\right\}$ in $\mathscr{I}$ such that $A_{j} \triangle B_{j}$ is a finite set for each $j \in \mathbb{N}$ and $B=\bigcup_{j=1}^{\infty} B_{j} \in \mathscr{I}$.

We prove that

$$
\begin{aligned}
& \lim _{\substack{m \rightarrow \infty \\
m \in M}} u_{m n} \\
& \quad=v_{n} \text { for some fuzzy numbers } v_{n} \text { and for each } n \in \mathbb{N}
\end{aligned}
$$

for $M=\mathbb{N} \backslash B \in \mathscr{F}(\mathscr{I})$. Let $\delta>0$ be given. Choose $k \in \mathbb{N}$ such that $1 / k<\delta$. Then, we have

$$
\begin{aligned}
& \left\{m \in \mathbb{N}: D\left(u_{m n}, v_{n}\right) \geq \delta\right\} \\
& \subset \bigcup_{j=1}^{k} A_{j} \text { for some fuzzy numbers } v_{n} \text { and for each } n \in \mathbb{N} .
\end{aligned}
$$

Since $A_{j} \triangle B_{j}$ is a finite set for $j \in\{1,2, \ldots, k\}$, there exists $m_{0} \in \mathbb{N}$ such that

$$
\left(\bigcup_{j=1}^{k} B_{j}\right) \cap\left\{m: m \geq m_{0}\right\}=\left(\bigcup_{j=1}^{k} A_{j}\right) \cap\left\{m: m \geq m_{0}\right\} .
$$

If $m \geq m_{0}$ and $m \notin B$ then

$$
m \notin \bigcup_{j=1}^{k} B_{j} \text { and so } m \notin \bigcup_{j=1}^{k} A_{j} \text {. }
$$

Thus, we have $D\left(u_{m n}, v_{n}\right)<1 / k<\delta$ for some fuzzy numbers $v_{n}$ and for each $n \in \mathbb{N}$. This implies that

$$
\lim _{\substack{m \rightarrow \infty \\ m \in M}} u_{m n}(x)=v_{n}
$$


Hence, we have

$$
\mathscr{I}^{*}-\lim _{m \rightarrow \infty} u_{m n}=v_{n}
$$

for some fuzzy numbers $v_{n}$ and for each $n \in \mathbb{N}$.

Similarly, for the set $C(\varepsilon)=\left\{n \in \mathbb{N}: D\left(u_{m n}, w_{m}\right) \geq \varepsilon\right\} \in$ $\mathscr{I}$, we have

$$
\mathscr{I}^{*}-\lim _{n \rightarrow \infty} u_{m n}=w_{m}
$$

for some fuzzy numbers $w_{m}$ and for each $m \in \mathbb{N}$. Hence, a double sequence $\left(u_{m n}\right)$ of fuzzy numbers is $r\left(\mathscr{I}_{2}^{*}, \mathscr{I}^{*}\right)$ convergent.

Now, we give the definitions of $r\left(\mathscr{I}_{2}, \mathscr{I}\right)$-Cauchy sequence and $r\left(\mathscr{I}_{2}^{*}, \mathscr{I}^{*}\right)$-Cauchy sequence.

Definition 14. Let $\mathscr{I}_{2}$ be a strongly admissible ideal of $\mathbb{N} \times \mathbb{N}$, and let $\mathscr{I}$ be an admissible ideal of $\mathbb{N}$. A double sequence $\left(u_{m n}\right)$ of fuzzy numbers is said to be regularly $\left(\mathscr{I}_{2}, \mathscr{I}\right)$-Cauchy $\left(r\left(\mathscr{I}_{2}, \mathscr{I}\right)\right.$-Cauchy), if it is $\mathscr{I}_{2}$-Cauchy in Pringsheim's sense and for every $\varepsilon>0$ there exist $k_{n}=k_{n}(\varepsilon) \epsilon$ $\mathbb{N}$ and $l_{m}=l_{m}(\varepsilon) \in \mathbb{N}$ such that the following statements hold:

$$
\begin{aligned}
& A_{1}(\varepsilon)=\left\{m \in \mathbb{N}: D\left(u_{m n}, u_{k_{n} n}\right) \geq \varepsilon\right\} \in \mathscr{I}, \quad(n \in \mathbb{N}), \\
& A_{2}(\varepsilon)=\left\{n \in \mathbb{N}: D\left(u_{m n}, u_{m l_{m}}\right) \geq \varepsilon\right\} \in \mathscr{I}, \quad(m \in \mathbb{N}) .
\end{aligned}
$$

A double sequence $\left(u_{m n}\right)$ is said to be regularly $\left(\mathscr{I}_{2}^{*}, \mathscr{I}^{*}\right)$ Cauchy $\left(r\left(\mathscr{I}_{2}^{*}, \mathscr{I}^{*}\right)\right.$-Cauchy), if there exist the sets $M \in$ $\mathscr{F}\left(\mathscr{I}_{2}\right), M_{1} \in \mathscr{F}(\mathscr{I})$, and $M_{2} \in \mathscr{F}(\mathscr{I})$ (i.e., $\mathbb{N} \times \mathbb{N} \backslash M \in \mathscr{I}_{2}$, $\mathbb{N} \backslash M_{1} \in \mathscr{I}$, and $\left.\mathbb{N} \backslash M_{2} \in \mathscr{I}\right)$ and for every $\varepsilon>0$ there exist $N=N(\varepsilon), s=s(\varepsilon), t=t(\varepsilon), k_{n}=k_{n}(\varepsilon), l_{m}=l_{m}(\varepsilon) \in \mathbb{N}$ such that

$$
\begin{aligned}
& D\left(u_{m n}, u_{s t}\right)<\varepsilon \text { for }(m, n),(s, t) \in M, \\
& D\left(u_{m n}, u_{k_{n} n}\right)<\varepsilon \text { for each } m \in M_{1} \text { and for each } n \in \mathbb{N}, \\
& D\left(u_{m n}, u_{m l_{m}}\right)<\varepsilon \text { for each } n \in M_{2} \text { and for each } m \in \mathbb{N},
\end{aligned}
$$

whenever $m, n, s, t, k_{n}, l_{m} \geq N$.

Theorem 15. Let $\mathscr{I}_{2}$ be a strongly admissible ideal of $\mathbb{N} \times \mathbb{N}$, and let $\mathscr{I}$ be an admissible ideal of $\mathbb{N}$. If a double sequence $\left(u_{m n}\right)$ of fuzzy numbers is $r\left(\mathscr{I}_{2}^{*}, \mathscr{I}^{*}\right)$-Cauchy, then it is $r\left(\mathscr{I}_{2}, \mathscr{I}\right)$-Cauchy.

Proof. Since a double sequence $\left(u_{m n}\right)$ of fuzzy numbers is $r\left(\mathscr{I}_{2}^{*}, \mathscr{I}^{*}\right)$-Cauchy, it is $\mathscr{I}_{2}^{*}$-Cauchy. We know that $\mathscr{I}_{2}^{*}$ Cauchy implies $\mathscr{I}_{2}$-Cauchy by Lemma 7 . Also, since the double sequence $\left(u_{m n}\right)$ of fuzzy numbers is $r\left(\mathscr{I}_{2}^{*}, \mathscr{I}^{*}\right)$ Cauchy so there exist the sets $M_{1}, M_{2} \in \mathscr{F}(\mathscr{I})$ and for every $\varepsilon>0$ there exist $k_{n}=k_{n}(\varepsilon), l_{m}=l_{m}(\varepsilon) \in \mathbb{N}$ such that

$$
\begin{aligned}
& D\left(u_{m n}, u_{k_{n} n}\right)<\varepsilon \text { for each } m \in M_{1} \text { and for each } n \in \mathbb{N}, \\
& D\left(u_{m n}, u_{m l_{m}}\right)<\varepsilon \text { for each } n \in M_{2} \text { and for each } m \in \mathbb{N}
\end{aligned}
$$

for $N=N(\varepsilon) \in \mathbb{N}$ and $m, n, k_{n}, l_{m} \geq N$. Therefore, for $H_{1}=$ $\mathbb{N} \backslash M_{1}, H_{2}=\mathbb{N} \backslash M_{2} \in \mathscr{I}$ we have

$$
\begin{aligned}
A_{1}(\varepsilon)= & \left\{m \in \mathbb{N}: D\left(u_{m n}, u_{k_{n} n}\right) \geq \varepsilon\right\} \\
& \subset H_{1} \cup\{1,2, \ldots, N-1\}, \quad(n \in \mathbb{N})
\end{aligned}
$$

for $m \in M_{1}$ and

$$
\begin{aligned}
A_{2}(\varepsilon)= & \left\{n \in \mathbb{N}: D\left(u_{m n}, u_{m l}\right) \geq \varepsilon\right\} \\
& \subset H_{2} \cup\{1,2, \ldots, N-1\}, \quad(m \in \mathbb{N})
\end{aligned}
$$

for $n \in M_{2}$. Since $\mathscr{I}$ is admissible ideal

$$
H_{1} \cup\{1,2, \ldots, N-1\} \in \mathscr{I}, \quad H_{2} \cup\{1,2, \ldots, N-1\} \in \mathscr{I} .
$$

Hence, we have $A_{1}(\varepsilon), A_{2}(\varepsilon) \in \mathscr{I}$ and $\left(u_{m n}\right)$ is $r\left(\mathscr{I}_{2}, \mathscr{I}\right)$ Cauchy.

Theorem 16. Let $\mathscr{I}_{2}$ be a strongly admissible ideal of $\mathbb{N} \times \mathbb{N}$, and let $\mathscr{I}$ be an admissible ideal of $\mathbb{N}$. If a double sequence $\left(u_{m n}\right)$ of fuzzy numbers is $r\left(\mathscr{I}_{2}, \mathscr{I}\right)$-convergent, then $\left(u_{m n}\right)$ is $r\left(\mathscr{I}_{2}, \mathscr{I}\right)$-Cauchy sequence.

Proof. Let $\left(u_{m n}\right)$ be a $r\left(\mathscr{I}_{2}, \mathscr{I}\right)$-convergent double sequence of fuzzy numbers. Then $\left(u_{m n}\right)$ is $\mathscr{I}_{2}$-convergent, and by Lemma 6 , it is $\mathscr{I}_{2}$-Cauchy sequence of fuzzy numbers. Also for every $\varepsilon>0$, we have

$$
A_{1}\left(\frac{\varepsilon}{2}\right)=\left\{m \in \mathbb{N}: D\left(u_{m n}, v_{n}\right) \geq \frac{\varepsilon}{2}\right\} \in \mathscr{I}
$$

for some fuzzy numbers $v_{n}$, for each $n \in \mathbb{N}$ and

$$
A_{2}\left(\frac{\varepsilon}{2}\right)=\left\{n \in \mathbb{N}: D\left(u_{m n}, w_{m}\right) \geq \frac{\varepsilon}{2}\right\} \in \mathscr{I}
$$

for some fuzzy numbers $w_{m}$, for each $m \in \mathbb{N}$. Since $\mathscr{I}$ is admissible ideal, the sets

$$
A_{1}^{c}\left(\frac{\varepsilon}{2}\right)=\left\{m \in \mathbb{N}: D\left(u_{m n}, v_{n}\right)<\frac{\varepsilon}{2}\right\}, \quad(n \in \mathbb{N})
$$

for some fuzzy numbers $v_{n}$ and

$$
A_{2}^{c}\left(\frac{\varepsilon}{2}\right)=\left\{n \in \mathbb{N}: D\left(u_{m n}, w_{m}\right)<\frac{\varepsilon}{2}\right\}, \quad(m \in \mathbb{N})
$$

for some fuzzy numbers $w_{m}$, are nonempty and belong to $\mathscr{F}(\mathscr{I})$. For $k_{n} \in A_{1}^{c}(\varepsilon / 2),\left(n \in \mathbb{N}\right.$ and $\left.k_{n}>0\right)$ we have $D\left(u_{k_{n} n}, v_{n}\right)<\varepsilon / 2$ for some fuzzy numbers $v_{n}$. Now, for $\varepsilon>0$ we define the set

$$
B_{1}(\varepsilon)=\left\{m \in \mathbb{N}: D\left(u_{m n}, u_{k_{n} n}\right) \geq \varepsilon\right\}, \quad(n \in \mathbb{N}),
$$

where $k_{n}=k_{n}(\varepsilon)$. Let $m \in B_{1}(\varepsilon)$. Then for $k_{n} \in A_{1}^{c}(\varepsilon / 2)$, $\left(n \in \mathbb{N}\right.$ and $\left.k_{n}>0\right)$ we have

$$
\begin{aligned}
\varepsilon \leq D\left(u_{m n}, u_{k_{n} n}\right) & \leq D\left(u_{m n}, v_{n}\right)+D\left(u_{k_{n} n}, v_{n}\right) \\
& <D\left(u_{m n}, v_{n}\right)+\frac{\varepsilon}{2}
\end{aligned}
$$


for some fuzzy numbers $v_{n}$. This shows that

$$
\frac{\varepsilon}{2}<D\left(u_{m n}, v_{n}\right) \text { and so } m \in A_{1}\left(\frac{\varepsilon}{2}\right) \text {. }
$$

Hence, we have $B_{1}(\varepsilon) \subset A_{1}(\varepsilon / 2)$.

Similarly, for $l_{m} \in A_{2}^{c}(\varepsilon / 2)\left(m \in \mathbb{N}\right.$ and $\left.l_{m}>0\right)$ we have

$$
D\left(u_{m l_{m}}, w_{m}\right)<\frac{\varepsilon}{2}, \quad(m \in \mathbb{N})
$$

for some fuzzy numbers $w_{m}$. Therefore, it can be seen that

$$
B_{2}(\varepsilon)=\left\{m \in \mathbb{N}: D\left(u_{m l_{m}}, w_{m}\right) \geq \varepsilon\right\} \subset A_{2}\left(\frac{\varepsilon}{2}\right) .
$$

Hence, we have $B_{1}(\varepsilon), B_{2}(\varepsilon) \in \mathscr{I}$. This shows that $\left(u_{m n}\right)$ is $r\left(\mathscr{I}_{2}, \mathscr{I}\right)$-Cauchy sequence of fuzzy numbers.

\section{Acknowledgment}

The authors would like to express their pleasure to the referees for making some useful remarks and helpful suggestions which improved the presentation of the paper.

\section{References}

[1] M. Matloka, "Sequences of fuzzy numbers," Busefal, vol. 28, pp. 28-37, 1986.

[2] S. Nanda, "On sequences of fuzzy numbers," Fuzzy Sets and Systems, vol. 33, no. 1, pp. 123-126, 1989.

[3] F. Nuray and E. Savaş, "Statistical convergence of sequences of fuzzy numbers," Mathematica Slovaca, vol. 45, no. 3, pp. 269273, 1995

[4] F. Nuray, "Lacunary statistical convergence of sequences of fuzzy numbers," Fuzzy Sets and Systems, vol. 99, no. 3, pp. 353355, 1998

[5] E. Savaş, "On statistically convergent sequences of fuzzy numbers," Information Sciences, vol. 137, no. 1-4, pp. 277-282, 2001.

[6] B. Bede and S. G. Gal, "Almost periodic fuzzy-number-valued functions," Fuzzy Sets and Systems, vol. 147, no. 3, pp. 385-403, 2004.

[7] R. Saadati, "On the I-fuzzy topological spaces," Chaos, Solitons and Fractals, vol. 37, no. 5, pp. 1419-1426, 2008.

[8] Ö. Talo and F. Başar, "Determination of the duals of classical sets of sequences of fuzzy numbers and related matrix transformations," Computers \& Mathematics with Applications, vol. 58, no. 4, pp. 717-733, 2009.

[9] P. Kostyrko, T. Šalát, and W. Wilczyński, "I-convergence," Real Analysis Exchange, vol. 26, no. 2, pp. 669-686, 2000.

[10] F. Nuray and W. H. Ruckle, "Generalized statistical convergence and convergence free spaces," Journal of Mathematical Analysis and Applications, vol. 245, no. 2, pp. 513-527, 2000.

[11] P. Das, P. Kostyrko, W. Wilczyński, and P. Malik, "I and $I^{*}$ convergence of double sequences," Mathematica Slovaca, vol. 58 , no. 5, pp. 605-620, 2008.

[12] M. Balcerzak, K. Dems, and A. Komisarski, "Statistical convergence and ideal convergence for sequences of functions," Journal of Mathematical Analysis and Applications, vol. 328, no. 1, pp. 715-729, 2007.
[13] A. Komisarski, "Pointwise $I$-convergence and $I$-convergence in measure of sequences of functions," Journal of Mathematical Analysis and Applications, vol. 340, no. 2, pp. 770-779, 2008.

[14] M. Mursaleen and A. Alotaibi, "On $I$-convergence in random 2-normed spaces," Mathematica Slovaca, vol. 61, no. 6, pp. 933940, 2011.

[15] M. Mursaleen and S. A. Mohiuddine, "On ideal convergence in probabilistic normed spaces," Mathematica Slovaca, vol. 62, no. 1, pp. 49-62, 2012.

[16] A. Şahiner, M. Gürdal, S. Saltan, and H. Gunawan, "Ideal convergence in 2-normed spaces," Taiwanese Journal of Mathematics, vol. 11, no. 5, pp. 1477-1484, 2007.

[17] P. Das and P. Malik, "On extremal I-limit points of double sequences," Tatra Mountains Mathematical Publications, vol. 40, pp. 91-102, 2008.

[18] K. Dems, “On I-cauchy sequences," Real Analysis Exchange, vol. 30, no. 1, pp. 123-128, 2004/2005.

[19] V. Kumar, "On $I$ and $I^{*}$-convergence of double sequences," Mathematical Communications, vol. 12, no. 2, pp. 171-181, 2007.

[20] T. Salát, B. C. Tripaty, and M. Ziman, "On I-convergence field," Italian Journal of Pure and Applied Mathematics, vol. 17, pp. 4554, 2005.

[21] B. Tripathy and B. C. Tripathy, "On I-convergent double sequences," Soochow Journal of Mathematics, vol. 31, no. 4, pp. 549-560, 2005.

[22] V. Kumar and K. Kumar, "On the ideal convergence of sequences of fuzzy numbers," Information Sciences, vol. 178, no. 24, pp. 4670-4678, 2008.

[23] M. Mursaleen, S. A. Mohiuddine, and O. H. H. Edely, "On the ideal convergence of double sequences in intuitionistic fuzzy normed spaces," Computers \& Mathematics with Applications, vol. 59, no. 2, pp. 603-611, 2010.

[24] E. Dündar and Ö. Talo, " $\mathscr{I}_{2}$-convergence of double sequences of fuzzy numbers," Iranian Journal of Fuzzy Systems. In press.

[25] E. Dündar and Ö. Talo, " $\mathscr{F}_{2}$-Cauchy double sequences of fuzzy numbers," General Mathematics Notes. In press.

[26] B. Altay and F. Başar, "Some new spaces of double sequences," Journal of Mathematical Analysis and Applications, vol. 309, no. 1, pp. 70-90, 2005.

[27] J.-x. Fang and H. Huang, "On the level convergence of a sequence of fuzzy numbers," Fuzzy Sets and Systems, vol. 147, no. 3, pp. 417-435, 2004.

[28] Mursaleen and O. H. H. Edely, "Statistical convergence of double sequences," Journal of Mathematical Analysis and Applications, vol. 288, no. 1, pp. 223-231, 2003.

[29] A. Pringsheim, "Zur Theorie der zweifach unendlichen Zahlenfolgen," Mathematische Annalen, vol. 53, no. 3, pp. 289-321, 1900.

[30] E. Savaș and Mursaleen, "On statistically convergent double sequences of fuzzy numbers," Information Sciences, vol. 162, no. 3-4, pp. 183-192, 2004.

[31] L. A. Zadeh, "Fuzzy sets," Information and Computation, vol. 8, pp. 338-353, 1965. 


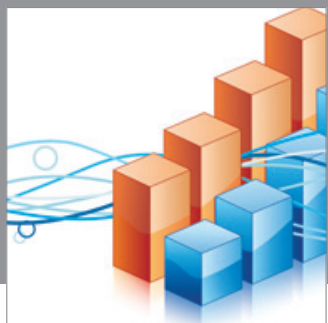

Advances in

Operations Research

mansans

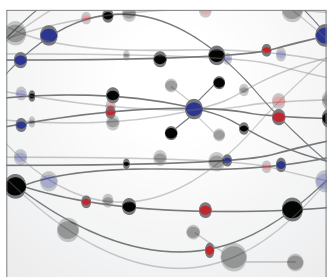

The Scientific World Journal
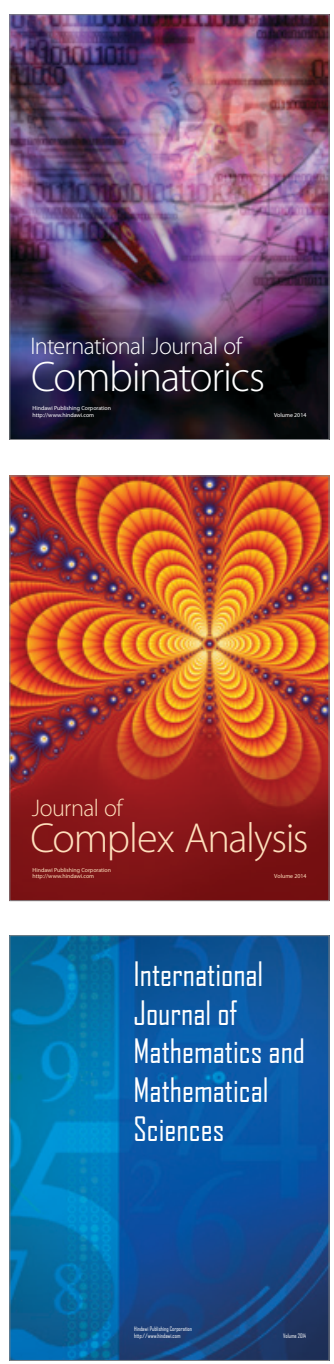
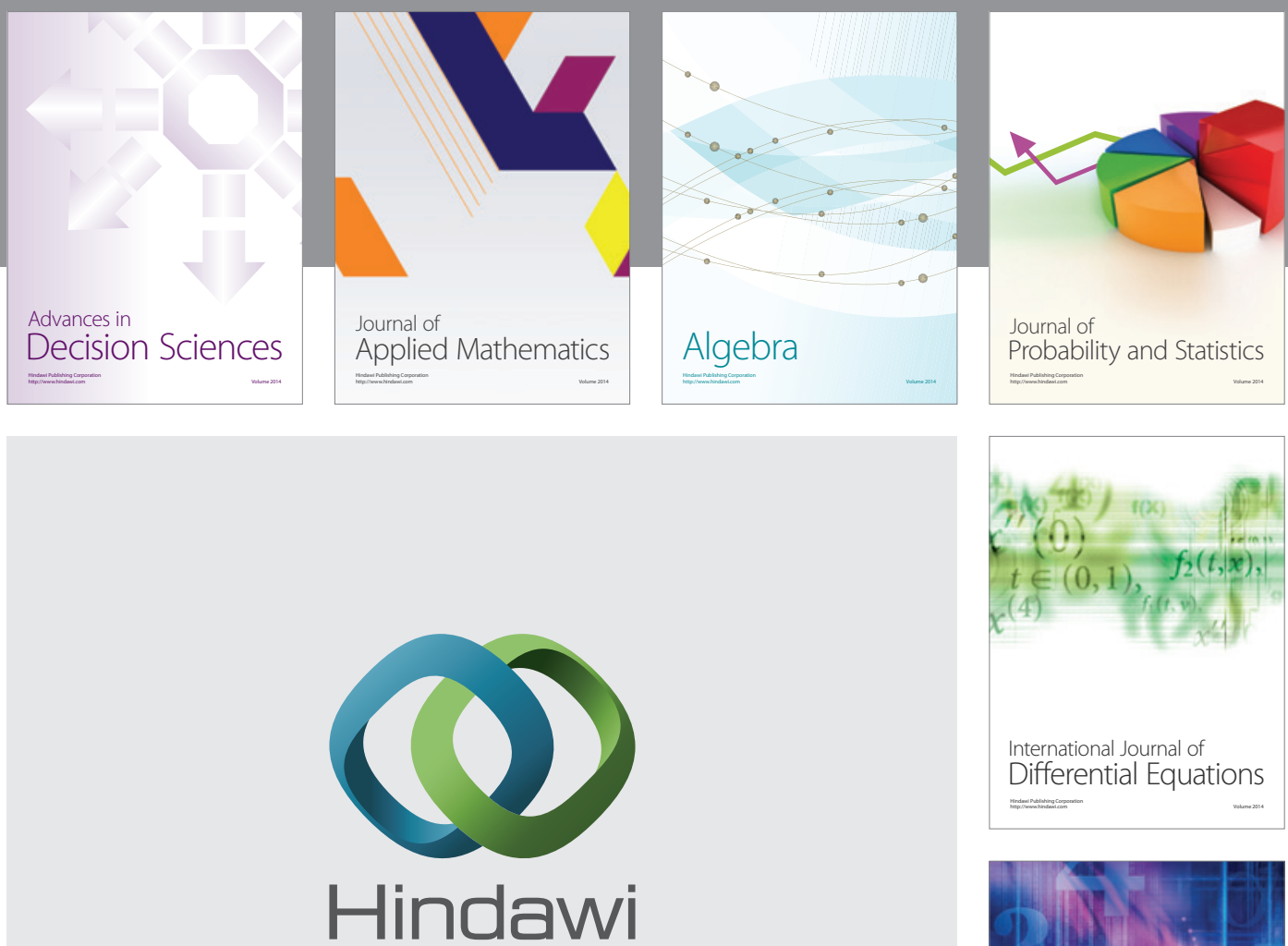

Submit your manuscripts at http://www.hindawi.com
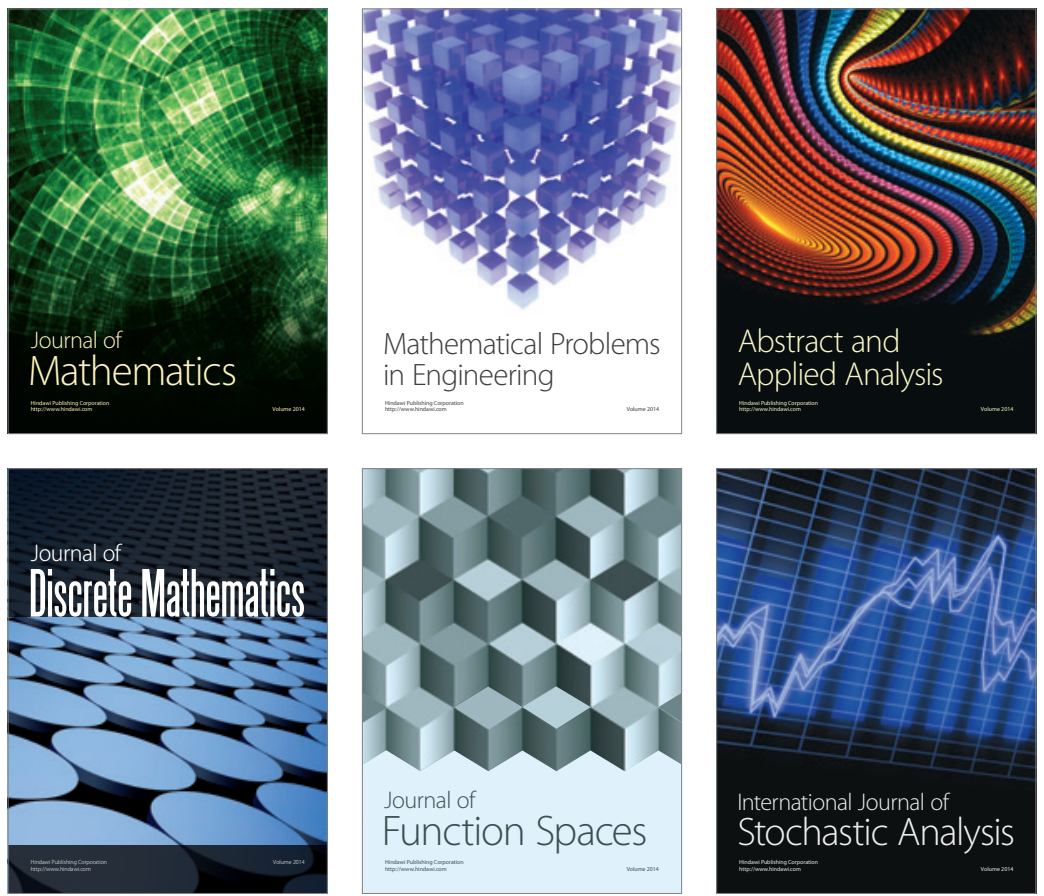

Journal of

Function Spaces

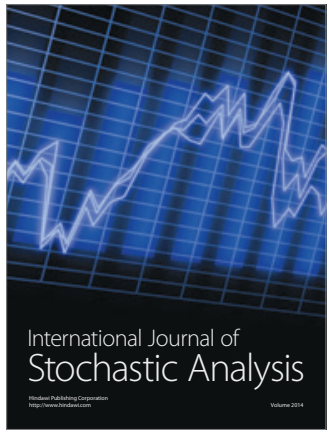

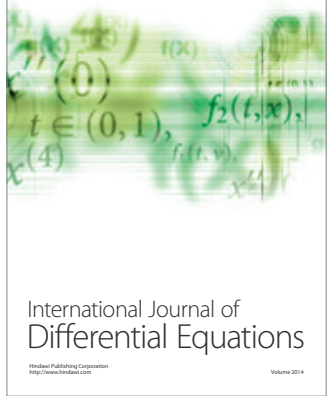
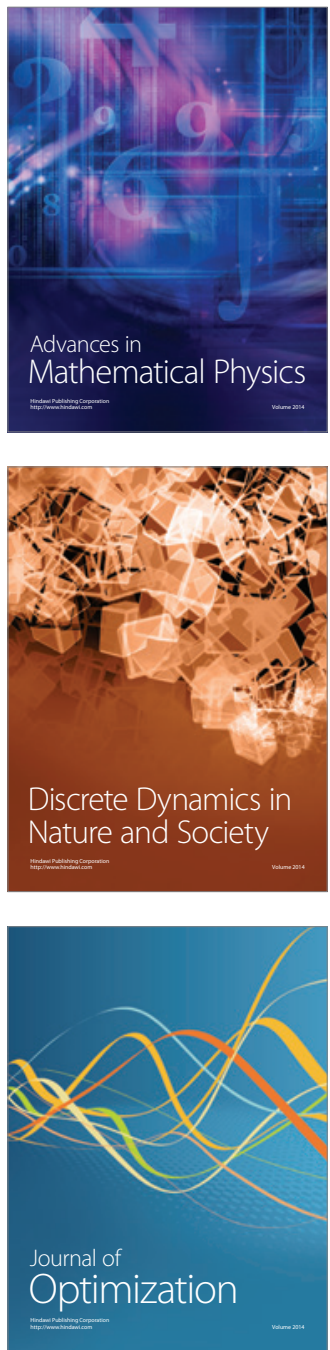